\title{
The Melanoma Genomics Managing Your Risk Study randomised controlled trial: statistical analysis plan
}

Serigne N. Lo ${ }^{1}$, Amelia K. Smit ${ }^{1,2,3}$, David Espinoza ${ }^{4}$, Anne E. Cust ${ }^{1,2^{*}}$ and on behalf of the Managing Your Risk Study Group

\begin{abstract}
Background: The Melanoma Genomics Managing Your Risk Study is a randomised controlled trial that aims to evaluate the efficacy of providing information on personal genomic risk of melanoma in reducing ultraviolet radiation (UV) exposure, stratified by traditional risk group (low or high phenotypic risk) in the general population. The primary outcome is objectively measured total daily Standard Erythemal Doses at 12 months. Secondary outcomes include UV exposure at specific time periods, self-reported sun protection and skin-examination behaviours, psychosocial outcomes, and ethical considerations surrounding offering genomic testing at a population level. A within-trial and modelled economic evaluation will be undertaken from an Australian health system perspective to assess the cost-effectiveness of the intervention.
\end{abstract}

Objective: To publish the pre-determined statistical analysis plan (SAP) before database lock and the start of analysis.

Methods: This SAP describes the data synthesis, analysis principles and statistical procedures for analysing the outcomes from this trial. The SAP was approved after closure of recruitment and before completion of patient followup. It outlines the planned primary analyses and a range of subgroup and sensitivity analyses. Health economic outcomes are not included in this plan but will be analysed separately. The SAP will be adhered to for the final data analysis of this trial to avoid potential analysis bias that may arise from knowledge of the outcome data.

Results: This SAP is consistent with best practice and should enable transparent reporting.

Conclusion: This SAP has been developed for the Melanoma Genomics Managing Your Risk Study and will be followed to ensure high-quality standards of internal validity and to minimise analysis bias.

Trial registration: Prospectively registered with the Australian New Zealand Clinical Trials Registry, ID: ACTR N12617000691347. Registered on 15 May 2017.

Keywords: Melanoma, Genomic risk, Behaviours, Prevention, Early detection, Randomised controlled trial, Sun exposure, Sun protection, Psycho-oncology, Bioethics

\footnotetext{
* Correspondence: anne.cust@sydney.edu.au

${ }^{1}$ The University of Sydney, Melanoma Institute Australia, Sydney, NSW,

Australia

${ }^{2}$ The University of Sydney, Faculty of Medicine and Health, Sydney School of

Public Health, Cancer Epidemiology and Prevention Research, Sydney, NSW,

Australia

Full list of author information is available at the end of the article
}

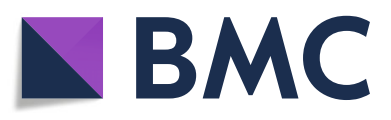

(C) The Author(s). 2020 Open Access This article is licensed under a Creative Commons Attribution 4.0 International License, which permits use, sharing, adaptation, distribution and reproduction in any medium or format, as long as you give appropriate credit to the original author(s) and the source, provide a link to the Creative Commons licence, and indicate if changes were made. The images or other third party material in this article are included in the article's Creative Commons licence, unless indicated otherwise in a credit line to the material. If material is not included in the article's Creative Commons licence and your intended use is not permitted by statutory regulation or exceeds the permitted use, you will need to obtain permission directly from the copyright holder. To view a copy of this licence, visit http://creativecommons.org/licenses/by/4.0/ The Creative Commons Public Domain Dedication waiver (http://creativecommons.org/publicdomain/zero/1.0/) applies to the data made available in this article, unless otherwise stated in a credit line to the data. 


\section{Introduction Preface}

Melanoma is a serious form of skin cancer associated with significant morbidity and mortality but is highly preventable through individual behaviour change $[1,2]$. Reduced sun exposure and improved primary prevention behaviours (i.e. sun protection) could prevent over $80 \%$ of melanoma diagnoses [3]. However, in high-incidence countries, such as Australia, preventive behaviours remain sub-optimal and there is a need to further improve melanoma prevention strategies aimed at the broader population [4].

Genomic risk information, based on multiple common genetic variants associated with elevated risk, could be integrated into improved melanoma prevention and early detection strategies $[5,6]$. Social and health behaviour theory suggests that personal genomic risk information, alongside support and education on prevention and early detection, may be more effective in motivating behaviour change than 'one size fits all' standard approaches [7-9]. But the research evidence on genomic risk interventions in healthy participants is limited by small sample sizes and a high risk of bias, and few of these studies have focussed on melanoma prevention behaviours $[10,11]$.

The Melanoma Genomics Managing Your Risk Study, an innovative randomised controlled trial (RCT), aims to establish whether providing personal genomic risk information modifies preventive behaviours, compared to receiving standard prevention advice [12]. A pilot RCT demonstrating feasibility and acceptability, and the trial protocol, were previously published $[12,13]$. The study was prospectively registered (ANCTRN 12615000356561). The trial completed the target accrual in February 2019, and all participants will be followed up for 12 months. The statistical analysis plan (SAP) reported here is Version 1.1, signed off by the principle investigator and study statisticians on 24 April 2020. The corresponding protocol has been published elsewhere [12]. The reporting of this SAP is in accordance with the guidelines for the content of SAPs in clinical trials [14].

\section{Purpose of the study and analyses}

To evaluate the impact of personal genomic risk of melanoma information on motivating skin cancer prevention behaviours, and on broader social, psychological, ethical and economic outcomes.

\section{Study aims and outcome measures \\ Aims \\ Primary}

The primary aim is to evaluate the efficacy of providing information on personal genomic risk of melanoma in reducing ultraviolet radiation (UV) exposure at 12 months, stratified by traditional risk group (low or high phenotypic risk) in the general population.

\section{Secondary}

The secondary aims are to evaluate:

1. The intervention's effect at 12 months on:
(a) time-specific UV exposure
(b) self-reported UV exposure
(c) sun-protection behaviours
(d) skin examinations

2. The effect on other behavioural outcomes including:

(a) tanning

(b) sunburn frequency

(c) hypothesised mediators of behaviour change

3. Psychological outcomes, including skin cancerrelated worry and distress

4. The intervention's effect on short-term outcomes at 1 month

5. The impact of personal genomic risk categories (lower than average, average or higher than average) on primary and secondary outcomes in the intervention arm alone

6. The experience of the intervention, psychological impact of undergoing genomic testing and receiving results, and communication with others about the results

Sub-studies beyond the scope of this SAP:

7. Qualitative sub-study assessing ethical considerations surrounding offering genomic testing at a population level, and psychosocial issues arising from the study processes that may affect wider implementation

8. Cost-effectiveness analysis of the intervention at 12 months and in the longer term from an Australian health-system perspective (refer to the Health Economics Analysis Plan (HEAP [15]) for more details)

9. Correlates of sun exposure, sun protection and skin examination behaviours, using baseline data

\section{Outcome measures}

\section{Primary outcome measure}

The primary outcome for this study is total daily Standard Erythemal Doses (SEDs) measured 12 months after the baseline assessment (baseline questionnaire and UV dosimeter). A SED is an objective measure of UV exposure, measured using a time-stamped electronic dosimeter badge, mounted in light-weight, custom-made wristbands attached to the left wrist (similar to wearing a watch) during daylight hours [16]. UV dosimetry is the gold standard for assessing personal UV exposure $[17,18]$. UV dosimeters 
will be worn by all participants for 10 days at baseline, and again 12 months after baseline. A subgroup of $\sim 240$ participants will also wear a UV dosimeter 1 month after receipt of the booklet/s. Total daily SEDs will be calculated as the weighted average of the average daily weekday and weekend SEDs (i.e. (weekday $\times 5+$ weekend $\times 2$ )/7). SED values will be log-transformed for all analyses.

\section{Secondary outcome measures}

Time-specific UV exposure captured from the timestamped UV dosimeters. This will be calculated as the weighted average within given time points: morning, peak and afternoon periods will be defined respectively as ' 6 a.m. to 10 a.m., 10 a.m. to 2 p.m. and 2 p.m. to 8 p.m.' (without daylight savings).

Self-reported UV exposure on weekdays and weekends assessed using questionnaire items: Thinking about the past month, please tell us the times of day as well as the usual length of time that you spent outside between 7 am and 6 pm on a typical weekday and weekend. Length of time is collected in $15 \mathrm{~min}$ intervals $(0,<15,15-29,30-44,45-60$ min), and will be recoded to the mid-point of the respective exposure times. Total weekday and weekend sun exposure will be calculated as the sum of mid-point exposure times. Self-reported UV exposure will be calculated as the weighted average of the average weekday and weekend self-reported UV exposure (i.e. (weekday $\times 5+$ weekend $\times 2$ ) $/ 7$ ).

Sun-protection behaviours [19] assessed individually and summarised as a Sun Protection Habits Index, which is calculated as the mean of six protective behaviours scored on a 4 -point Likert scale $(1=$ never or rarely, $2=$ sometimes, $3=$ often, $4=$ always): During the past month, when outside, how often did you...

(a) Wear sunscreen?

(b) Wear a shirt with sleeves that cover your shoulders?

(c) Wear a hat?

(d) Stay in the shade or under an umbrella?

(e) Wear sunglasses?

(f) Limit your time in the sun during midday hours?

Sunscreen use is assessed using an item: Have you used sunscreen in the past month? (yes/no). If participants respond 'yes' they are asked the following items:

- Was this usually a high-protection sunscreen (SPF 30 or more)? (yes/no)

- How often on average have you used sunscreen in the past month? (less than once a week, 1-2 days a week, 3-5 days a week, 6-7 days a week)

- On days that you have used sunscreen in the past month, how often did you apply it? (open response restricted to numbers)
- Did you usually apply sunscreen (select one of the following): to all parts of your body exposed to the sun OR only to parts of your body that are prone to sunburn.

Hat wear is assessed an additional item: Please select the option which is most similar to your usual headwear when in the sun in the past month. Response options are: no headwear, beanie, cap, legionnaire's hat, bucket hat, wide-brimmed hat, veil/burka.

Whole-body skin examinations, performed on oneself or by a partner or (in a separate question) by a health professional $[19,20]$ will be dichotomised as 'yes' vs. 'no'. If the response in the 12-month questionnaire to either:

(a) In the past 12 months, have you, your partner, friend or family member checked at least some of your skin for any suspicious spots that might be skin cancer? or

(b) In the past 12 months, have you had a health professional (e.g. a physician, specialist or nurse) check at least some of your skin for any suspicious spots that might be skin cancer?

is 'yes' then the extent of skin check $(1=$ all or nearly all of your body, 2 = part of your body, 3 = checking a specific mole or spot) is assessed. If either the self-check or health professional check response is 'All or nearly all of your body' then whole skin examinations will be coded as 1: yes. All other responses (including those that have no skin checks) will be coded as 0: no.

Intentional tanning frequency will be measured on a 5 -point Likert scale $(1=$ never, 2 = rarely, 3 = sometimes; $4=$ often, $5=$ always) in response to the question: In the past month, how often do you spend time in the sun in order to get a tan?

Sunburn frequency over the previous month [20] will be collected as ' 0 ', ' 1 ', ' 2 ', or ' 3 or more times' in response to the question: In the past month, how many times did you have a red or painful sunburn that lasted a day or more? This will also be categorised as 'any' versus 'none'.

Hypothesised mediators of behaviour change will be measured by the following items:

Perceived severity of melanoma [21]: To what extent do you agree or disagree with the following?

- I believe that melanoma is easy to cure

- I believe that melanoma can have very severe consequences

- Getting melanoma would be a big health threat for me

A mean score will be derived from response options on a 5-point Likert scale: 1 = strongly disagree, 2 = disagree, 
$3=$ neither agree nor disagree, $4=$ agree, $5=$ strongly agree.

Perceived risk of melanoma [22]:

Write a number between 0 and 100 (where 0 means no chance and 100 means absolute certainty) to show what you think the chance is that you will develop melanoma during your remaining lifetime.

How confident are you in the number you wrote above? Response options are on a 5-point Likert scale: $1=$ not at all confident, $2=$ slightly confident, $3=$ somewhat confident, $4=$ quite confident, $5=$ very confident [23].

Compared to the average person of your sex and age, what do you think the chance is that you will develop melanoma in your lifetime? Response options are on a 5-point Likert scale: $1=$ much below average, $2=$ somewhat below average, $3=$ average, $4=$ above average, $5=$ much above average.

Confidence in identifying melanoma [21]; How confident are you in your ability to identify melanoma? Response options are on a 5-point Likert scale: $1=$ not at all confident, $2=$ slightly confident, $3=$ somewhat confident, 4 = quite confident, 5 = very confident.

Perceived effectiveness of specific sun-protection behaviours in reducing personal melanoma risk [24]: For me, (using sunscreen/wearing protective clothing (such as long sleeves, long pants, hat)/avoiding sun exposure during midday hours) is (or would be) effective in reducing my risk of developing melanoma. Response options for these three items are on a 5-point Likert scale: $1=$ not at all effective, $2=$ a little effective, $3=$ somewhat effective, $4=$ moderately effective, $5=$ very effective.

Perceived importance of protective behaviours: How important are these activities? Limiting sun exposure in the middle of the day; Wearing long-sleeved shirts; Wearing long pants; Wearing a wide-brimmed hat; Using sunscreen of SPF 30 or higher; Asking your physician to conduct a skin examination; Checking your own skin for suspicious spots/moles; and Staying in the shade. Response options are on a 4-point Likert scale: $1=$ not at all important, $2=$ not very important, $3=$ somewhat important, $4=$ extremely important.

Perceived capability of performing protective behaviours: How capable do you feel that you can do these things well, whether or not you intend to do them? (the same eight protective behaviours listed for perceived importance of protective behaviours above). Response options are on a 4point Likert scale: $1=$ not at all capable, $2=$ not very capable, 3 = somewhat capable, 4 = extremely capable.

Social norms about skin examinations and sun protection [21]: How many (adult members of your family/ friends) check their own skin, or have their skin checked by someone else at least every 2 years? How many of your (family members/friends) do you think regularly take precautions in the sun (such as using sunscreen, wearing protective clothing, avoiding sun during midday hours)? Response options are: $1=$ none, $2=$ some, $3=$ half, $4=$ most, 5 = all, 6 = I don't know.

Skin Self-Examination Attitude Scale (SSEAS) [25]: To what extent do you agree with the following statements:

- It is important to check my skin for skin cancer even if I have no symptoms

- Checking my skin regularly is a priority for me

- I think I could find something suspicious on my skin if it was there

- If I saw something suspicious on my skin, I'd go to the physician straight away

- I am confident in a physician's ability to diagnose skin cancer

- I am confident that I can take up examining my own skin even if I have not looked at my skin the past few months

- I am able to examine my own skin regularly, even if I have no one to help me

- If I regularly examine my skin, then I am helping to look after my own health

Response options are on a 5-point Likert scale: $0=$ strongly disagree, $1=$ disagree, $2=$ unsure, $3=$ agree, $4=$ strongly agree. Responses to each item will be summated to generate a total score that can range between 0 and 40.

Tanning attitudes (pro-tan score) [26]: To what extent do you agree with the following statements:

- I feel more healthy with a suntan

- A suntan makes me feel better about myself

- A suntan makes me feel more attractive to others

- This summer I intend to sunbathe regularly to get a suntan

- Most of my close family think that a suntan is a good thing

- Most of my friends think a suntan is a good thing

- A suntan protects you against melanoma and other skin cancers

Response options are on a 5-point Likert scale: $1=$ strongly disagree, $2=$ disagree, $3=$ neither agree nor disagree, $4=$ agree, $5=$ strongly agree. Responses to the seven items will be summated, giving a total score between 7 and 35; those with a total score of 7-14 will be classified as 'anti-tan'.

Anxiety about skin-examinations [25]: I think checking my skin would make me anxious. Response options are on a 5 -point Likert scale: $1=$ strongly disagree, $2=$ disagree, $3=$ unsure, 4 = agree, $5=$ strongly agree.

Perceived control over developing melanoma [24]; Overall, how much personal control do you feel you have 
over (whether you develop melanoma in the future?/detecting a future melanoma early in its development?) Response options are on a 5-point Likert scale: $1=$ no control, $2=$ not much control, $3=$ some control, $4=$ moderate control, $5=$ a lot of control.

Family communication: In the past month, how frequently have you discussed with your family: using sunscreen; wearing protective clothing (such as long sleeves, long pants, hat); avoiding sun exposure during midday hours; getting a skin check by a health professional (e.g. general practitioner (GP) or dermatologist); doing personal skin checks; spending time in the sun to get a tan? Response options are on a 5-point Likert scale: $1=$ not at all, $2=$ a little, $3=$ sometimes, $4=$ quite a bit, $5=$ a lot.

Vitamin D knowledge: Do you believe that if you always protect all your skin from the sun, you are in danger of not getting enough Vitamin D? Response options are: yes, no, I don't know.

Health professional advice about sun protection and skin examinations: In the past 12 months, has a health professional provided you with information or advice about sun protection, or how to check your skin for early signs of melanoma? Response options are: yes, no.

Barriers to sun protection: If you do not regularly use sunscreen, can you tell us the reason for this? If you do not regularly wear protective clothing (such as long sleeves, long pants, hat), can you tell us the reason for this? If you do not regularly limit your time in the sun during midday hours, can you tell us the reason for this? Each of these items have a free-text response field and will be analysed using content and thematic analysis.

Other potential mediators include items on: the importance of personal health in achieving life goals [27] (assessed using a 5-point Likert scale); perceived general health [28] (assessed using a 5-point Likert scale); frequency of information-seeking related to skin cancer, genetics and genetic counselling; use of the study website; and use of applications (apps) to assist with managing sun protection or skin examinations.

\section{Psychological outcomes}

Melanoma-related worry will be assessed using three items:

(a) How worried are you about getting melanoma someday?

(b) How often does your worry affect your mood?

(c) How often does your worry affect your ability to perform your daily activities?

Each item will be assessed on a 5-point Likert scale: $1=$ not at all, $2=$ rarely, $3=$ sometimes, $4=$ often, $5=$ al most all the time, and a mean score will be calculated.
Psychological distress and well-being will be assessed separately using the five-item version of the Mental Health Inventory (MHI-5) [29]. The MHI-5 is a subscale of the 36-item Short Form Health Survey (SF-36) comprising the following items: How much of the time during the past month have you:

(a) Been a very nervous person?

(b) Felt so down in the dumps that nothing could cheer you up?

(c) Felt calm and peaceful?

(d) Felt downhearted and blue?

(e) Been a happy person?

Response options are on a 6-point Likert scale ranging from $1=$ never to $6=$ all of the time. Items (c) and (e) above will be reverse-coded and the five scores then summated to a total subscale score in which higher scores indicate a worse health state and lower scores a better health state. Up to two missing values on the subscale will be imputed using the personal mean values from the completed items; otherwise the total score will be set to missing [30]. Each raw scale subscale score (ranging from 5 to 30) will be transformed to a 0 to 100 scale using the formula below, where the lowest possible raw score is 5 and the possible raw score range is 25 :

Transformed Scale $=\frac{(\text { Actual raw score-Lowest possible raw score })}{\text { Possible raw score range }} \times 100$.

Intervention arm additional measures: the experience of the intervention, the psychological impact of undergoing genomic testing and receiving the results, and communication with others about the results, will be assessed using several items.

The psychological impact of undergoing testing and receiving personal genomic risk information will be assessed through the Multidimensional Impact of Cancer Risk Assessment (MICRA) [31]. The MICRA consists of three subscales (a) distress (six items), (b) uncertainty (nine items) and (c) positive experiences (four items - reverse scaled) (Table 1).

Additional MICRA items that are assessed separately to the subscales include: Feeling regret about getting my risk information; and Understanding clearly my choices for melanoma prevention or early detection. In addition, if the participant has a child(ren), two additional items are assessed: Worrying about the possibility of my chil$\mathrm{d}(\mathrm{ren})$ getting melanoma; Feeling guilty about possibly passing on the melanoma risk to my child(ren). If the participant has cancer, or has had it in the past, the following items are also assessed: Feeling that my genetic risk information has made it harder to cope with my cancer; and Feeling that my genetic risk information has made it easier to cope with my cancer. The responses 
Table 1 Multidimensional Impact of Cancer Risk Assessment (MICRA) domain subscales

\begin{tabular}{|c|c|}
\hline \multirow[t]{6}{*}{ Distress (6 items) } & Feeling upset about my risk information \\
\hline & Feeling sad about my risk information \\
\hline & Feeling anxious or nervous about my risk information \\
\hline & Feeling guilty about my risk information \\
\hline & Feeling a loss of control \\
\hline & Having problems enjoying life because of my risk information \\
\hline \multirow[t]{9}{*}{ Uncertainty (9 items) } & $\begin{array}{l}\text { Worrying about my risk of getting cancer (or getting cancer again if you have already } \\
\text { been diagnosed with cancer) }\end{array}$ \\
\hline & Being uncertain about what my risk information means about my cancer risk \\
\hline & $\begin{array}{l}\text { Being uncertain about what my risk information means for my child(ren) and/or } \\
\text { family's cancer risk }\end{array}$ \\
\hline & $\begin{array}{l}\text { Having difficulty making decisions about cancer screening or prevention (e.g. having } \\
\text { preventive surgery or getting medical tests done) }\end{array}$ \\
\hline & Feeling frustrated that there are no definite cancer prevention guidelines for me \\
\hline & Thinking about my risk information has affected my work or family life \\
\hline & Feeling concerned about how my risk information will affect my insurance status \\
\hline & Having difficulty talking about my risk information with family members \\
\hline & $\begin{array}{l}\text { Worrying that the genetic counselling and testing process has brought about conflict } \\
\text { within my family }\end{array}$ \\
\hline \multirow[t]{4}{*}{ Positive experiences ( 4 items - reverse scaled) } & Feeling relieved about my risk information \\
\hline & Feeling happy about my risk information \\
\hline & Feeling that my family has been supportive during the genetic counselling and testing process \\
\hline & Feeling satisfied with family communication about my genetic risk information \\
\hline
\end{tabular}

are scored as 0 for 'never', 1 for 'rarely', 3 for 'sometimes' and 5 for 'often' for the distress and uncertainty MICRA subscale items; and as 5 for 'never', 3 for 'rarely', 1 for 'sometimes' and 0 for 'often' for the positive experiences subscale items. The scores will be summated for each subscale, and the three subscales also summated to give a total score. Higher values of the MICRA score are related to increased experiences of distress. The questionnaire items for the additional MICRA items will be scored the same way as the distress and uncertainty subscales, with the possible range of $0-5$ when there is one item, and $0-10$ when there are two items.

The Genetic Counselling Satisfaction Survey (GCSS) [32]: To what extent do you agree with the following statements?

- I felt I could talk about my reaction to my risk information with the genetic counsellor

- The genetic counsellor helped me to understand my risk information and make decisions about my health care

- I felt better about my health after talking to the genetic counsellor

- The length of the phone call was appropriate
- The genetic counsellor was truly concerned about my well being

- Talking to the genetic counsellor was valuable to me

- Understanding of genetic risk information, and amount read by participants

Response options are on a 5-point Likert scale: $1=$ strongly disagree, 2 = disagree somewhat, $3=$ uncertain, $4=$ agree somewhat, 5 = agree strongly.

- Recall of personal genetic risk category

- Communication of result with family, friends and health professionals

- Motivations and barriers to family communication about genetic risk [33]

- Satisfaction, understanding and amount read of the personal genetic risk booklet

\section{Methods}

General study design and plan

The Melanoma Genomics Managing Your Risk Study is a parallel-group, two-arm, RCT with a 1:1 allocation ratio. Supplementary Figure 1 shows the schema for the trial. 


\section{Eligibility criteria}

Eligible participants met all of the following criteria:

1. Aged 18-69 years at the time of recruitment

2. Never had a melanoma

3. Some European ancestry, since current knowledge of genomic variation associated with melanoma risk is based predominately on populations with European ancestry, although there are ongoing efforts to improve this disparity [34]

\section{Randomisation and blinding}

Randomisation occurred once participants had completed their baseline questionnaire and returned their baseline UV dosimeter. Randomisation to the intervention or control arm (allocation ratio 1:1) was conducted independently by the University of Sydney's NHMRC Clinical Trials Centre Randomisation Service using a computer-based system. The stratified minimisation procedure [35] was used to ensure that the study groups were balanced by:

- Traditional phenotypic risk score (low vs. high) [36],

- Sex (male vs. female),

- State or territory of residence within Australia, and

- Age group (18-44 years vs. 45-69 years)

Traditional risk scores were classified as high or low based on a validated published risk prediction model that includes variables for moles (naevi), hair colour, eye colour, artificial sunbed use, first-degree family history of melanoma, and personal history of keratinocyte cancers (e.g. basal cell carcinoma, squamous cell carcinoma) [36]. For the traditional risk score, a cut-point of 1.223 was used to ensure that approximately half of participants were in the low/high-risk groups; this cut-point is based on data from our pilot trial and Australian Melanoma Family Study controls) [13, 36].

\section{Study variables}

Outcomes will be measured at baseline, 1 month after receipt of the booklet/s (except dosimeter outcomes that were only assessed for a subset of participants at 1 month), and again 12 months after baseline as summarised in Table 2. A sample of 240 participants will wear a UV dosimeter for 10 days at follow-up 1 ( 1 month after receipt of booklet/s).

\section{Sample size}

Sample size calculations are based on detecting a 20\% difference in the primary outcome of average daily SEDs between intervention and control arms, in each of the low- and the high-risk phenotype groups. There is currently no consensus as to a 'safe' daily SED limit. Instead, skin cancer prevention strategies for the public primarily aim to shift the distribution of sun exposures downwards, especially in seasons of peak exposure. Due to the skewed nature of the SED exposure data, the sample size was calculated using a $t$ test with a geometric mean ratio (geometric mean SEDs in intervention group/geometric mean SEDs in control group) of 0.8, coefficient of variation $0.9,80 \%$ power and $\alpha$ of 0.05 .

Based on these calculations, a sample of 756 people (378 in the high-risk phenotype group and 378 in the low-risk phenotype group), split evenly between the intervention and control arms, will provide $80 \%$ power to detect a $20 \%$ reduction in the primary outcome in favour of the intervention with either low- or high-risk phenotypes. Allowing up to $15 \%$ with incomplete followup at 12 months, we will need to recruit 892 people (446 in each of intervention and control arms). Secondary analysis pooling the data from the low- and high-risk phenotype groups will give $>97 \%$ power to detect a $20 \%$ difference in SEDs between the intervention and control groups.

No interim analysis, safety analysis and or data monitoring is planned for this study due to the minimal risks associated with the intervention.

\section{General considerations for statistical analysis}

The analysis principles are as follows:

- All analyses will be conducted on an intention-to treat basis. All randomised participants will be analysed in the group to which they were assigned

- The primary analyses will stratify by phenotypic risk category (high, low)

- Statistical hypothesis tests will be evaluated at a nominal two-sided 5\% level of significance

- Intervention effect estimates (i.e. difference in means, odds ratio or relative risk) and their $95 \%$ confidence interval (CI) will be reported for all outcomes

- The assessment of the overall intervention effect on outcome measures will be adjusted for baseline scores and randomised stratification factors

- Subgroup analyses will be carried out irrespective of whether there is a significant effect of intervention on outcome

- $P$ values will not be adjusted for multiple comparisons.

- $P$ values will be reported to three decimal places unless the $P$ value is less than 0.001 , in which case it will be reported as ' $<0.001$ '. The mean, standard deviation (SD) and any other statistics other than quantiles will be reported to one decimal place greater than the original data. Quantiles, such as 
Table 2 Schedule of study parameters and collection time points

\begin{tabular}{|c|c|c|c|c|}
\hline \multirow[b]{3}{*}{ Time point } & \multicolumn{4}{|c|}{ Study period } \\
\hline & $\begin{array}{l}\text { Screening/ } \\
\text { consent }\end{array}$ & $\begin{array}{l}\text { Baseline/ } \\
\text { randomisation }\end{array}$ & $\begin{array}{l}\text { Intervention } \\
\text { delivery }\end{array}$ & Follow-up ${ }^{a}$ \\
\hline & -1 & 0 & $T 1$ & $F 1$ \\
\hline
\end{tabular}

\section{Enrolment:}

Eligibility screen

Informed consent

Allocation

\section{Group allocation:}

\section{Intervention arm:}

Saliva sample

Personalised melanoma genetic risk booklet

Phone call from genetic counsellor

Educational booklet on melanoma preventive behaviours

\section{Control arm:}

Educational booklet on melanoma preventive behaviours

\section{$x$}

Assessments (control and intervention patients):

Demographics

Melanoma risk factors

Sun exposure (objective measure)

Sun exposure (self-report)

Sun-protection behaviours

Skin examination behaviours

Intentions, beliefs and attitudes towards sun-protection behaviours and skin examinations

Discussion about sun protection and skin examinations with family

Perceived social norms about sun protection and skin examination

Perceived melanoma risk

Skin-cancer related worry

Perceived control over the development and early detection of future melanomas

Risk taking behaviours (Domain-Specific Risk-taking scale (DOSPERT))

Psychological distress and well-being (MHI-5)

$x$

Confidence in completing medical forms

General health

Satisfaction with educational booklet on melanoma preventive behaviours, and amount read by participants

Out-of-pocket costs for sun-protection items

Visits to health care professionals

Private health insurance

Use of medications that may increase risk of melanoma or skin

Confidence in understanding medical information 
Table 2 Schedule of study parameters and collection time points (Continued)

\begin{tabular}{|c|c|c|c|c|c|}
\hline \multirow[b]{3}{*}{ Time point } & \multicolumn{5}{|c|}{ Study period } \\
\hline & \multirow{2}{*}{$\begin{array}{l}\text { Screening/ } \\
\text { consent } \\
-1\end{array}$} & \multirow{2}{*}{$\begin{array}{l}\text { Baseline/ } \\
\text { randomisation } \\
0\end{array}$} & \multirow{2}{*}{$\begin{array}{l}\text { Intervention } \\
\text { delivery } \\
\text { T1 }\end{array}$} & \multicolumn{2}{|c|}{ Follow-up ${ }^{a}$} \\
\hline & & & & F1 & $F 2$ \\
\hline Information seeking about skin cancer and genetics & & $x$ & & $x$ & $x$ \\
\hline Use of the study website & & $x$ & & $x$ & $x$ \\
\hline Use of applications related to sun protection or skin examinations & & & & & $x$ \\
\hline \multicolumn{6}{|c|}{ Measures related to receiving genetic risk information (intervention participants only): } \\
\hline Recalling personal genetic risk & & & & $x$ & $x$ \\
\hline $\begin{array}{l}\text { Communication with family, friends and health professionals about } \\
\text { genetic risk }\end{array}$ & & & & $x$ & $x$ \\
\hline Motivation and barriers to communication about genetic risk & & & & $x$ & $x$ \\
\hline Satisfaction with genetic risk booklet and genetic counselling & & & & $x$ & $x$ \\
\hline $\begin{array}{l}\text { Understanding of genetic risk information, and amount read } \\
\text { by participants }\end{array}$ & & & & $x$ & $x$ \\
\hline Multidimensional impact of cancer risk assessment & & & & $x$ & $x$ \\
\hline
\end{tabular}

${ }^{\text {a}}$ Follow-up 1 (F1) takes place 1 month after participants receive their booklets. Follow-up 2 (F2) takes place 12 months after participants complete the baseline assessment (baseline questionnaire and ultraviolet radiation (UV) dosimeter)

${ }^{\mathrm{b}}$ The UV dosimeter measurement will be measured in $~ 240$ participants rather than in all participants at the short-term (1-month) follow-up

median, or minimum and maximum will use the same number of decimal places as the original data. Estimated parameters, not on the same scale as raw observations (e.g. regression coefficients), will be reported to three significant figures

- Analyses will be conducted primarily using SAS, version 9.4 or later and R 3.6.1 or later

\section{Timing of analyses}

The final analysis will be performed after all randomised participants have completed their 12-month follow-up (or dropped out prior to this).

\section{Analysis populations}

The final analysis will include all participants who were randomised. The analysis population will be split into one of two phenotypic risk groups (i.e. high-risk and low-risk) as per the randomisation stratification variable 'Traditional phenotypic risk score'. For secondary analysis of the primary outcome the analysis population will comprise the pooled phenotype risk groups.

\section{Covariates and subgroups}

The following subgroup analyses will be performed in each phenotypic risk groups and overall. It is hypothesised that the effect of the intervention on behaviour change or psycho-social outcomes may be influenced (moderated) by:

- Sex: male/other vs. female
- Age: 18-44 vs. 45-69 years

- State or territory of residence (based on latitude): QLD, NT, WA, NSW, ACT vs. SA, VIC, TAS

- Health literacy and numeracy: higher vs. lower [28]

- Family history of melanoma or a personal history of non-melanoma skin cancer: yes vs. no

- Education: school-only vs. higher education

- Socio-Economic Indexes for Areas (SEIFA) using the Index of Relative Socio-Economic Advantage and Disadvantage (IRSAD) based on postcode of residence and categorised into five groups using quintile cut-points

- Children: yes vs. no

- Risk taking propensity: risk-averse vs. risk-seeking (see the Domain-specific Risk-taking (DOSPERT) scale below [37])

- Taking medications such as immuno-suppressants that may increase their sensitivity to sunlight (yes, no, don't know)

- Genetic determinism, measured as How much do you think genetic make-up, that is characteristics that are passed down from one generation to the next, determine whether or not a person will develop melanoma? [38] on a 5-point Likert scale and categorised as 4 or 5 (completely/moderately) vs. 1, 2, or 3 (not at all, slightly, somewhat)

- Genomic risk category (available for the intervention group only): higher than average vs. average vs. lower than average risk

- Discordant genotype/phenotype groups (available for the intervention group only):

low-risk phenotype but high-risk genotype 
high-risk phenotype but low-risk genotype concordant combinations

DOSPERT: The Domain-specific Risk-taking (DOSPERT) [37] scale contains three separate constructs: 'risk-taking', 'risk-perceptions' and 'risk-attitude' across separate domains. Two domains of risk, 'Health and Safety' and 'Social' were shown to be relevant to a preventive intervention for sun-protection behaviours in our pilot study [39] and were included in the baseline questionnaire; the items are shown in Table 3. For the assessment of:

- Risk-taking - participants were asked to indicate 'The likelihood that you would engage in the described activity', from extremely unlikely to extremely likely on a 7-point scale

- Risk perception - participants were asked to indicate 'How risky you perceive the described situations' from not at all risky to extremely risky on a 7-point scale

- Risk attitude - participants were asked to indicate "The benefits that you would obtain from each situation', from no benefits at all to great benefits

Item responses will be summated to obtain subscale scores for each domain for 'risk-taking', 'risk-perceptions' and 'risk-attitude'. Individuals will be classified as 'risk seeking' if their subscale score was more than $1 \mathrm{SD}$ above the mean; 'risk averse' if their subscale score was more than $1 \mathrm{SD}$ below the mean; 'risk neutral' if their subscale score was within $1 \mathrm{SD}$ of the mean.

\section{Missing data}

The number of participants who complete the 12-month follow-up will be described by allocation; the study arms will be compared using chi-square tests and logistic regression to see if the attrition rate differs by arm and to compare baseline characteristics of participants who did and did not complete follow-up. Of those who complete follow-up, each variable will be examined for the presence of missing data and if $>10 \%$ is observed for primary or secondary outcomes, then sensitivity analyses will be performed using complete case analysis or multiple imputation methods assuming data are missing at random (MAR). The MAR assumption indicates that the propensity for missingness does not depend on the unobserved outcome but rather is related to some other observed data.

The distribution of dosimeter sun exposure days will be described by study arm. Non-adherence will be defined as participants that fail to wear the dosimeter over at least one day on both a weekday and a weekend. Level of adherence (e.g. number of days worn) will also be described. For participants who do not have UV dosimeter (SED) data for both weekday and weekend exposures, we will estimate their missing exposure using imputation methods based on the participant's available weekday or weekend SEDs, and SEDs data from the same age-group, gender, traditional (phenotypic) risk group and state or territory.

\section{Multiple testing}

There will be no formal adjustment for multiple comparisons. Outcomes are clearly categorised by degree of importance (primary and secondary) and cover a broad range of disciplines (e.g. behavioural, psychological, ethical, economic). Subgroup analyses have been prespecified and are based on strong rationale and behaviour change theory.

\section{Summary of study data}

Descriptive statistics will be prepared to summarise the data distributions and the characteristics of the study participants by allocation group. Continuous variables will be summarised using: $n$ (non-missing sample size), mean (SD), median (25th-75th centiles) and categorical variables will be summarised by frequency and percentages (based on the non-missing sample size) of the observed levels.

\section{Subject disposition}

Subject disposition will be summarised with a Consolidated Standards of Reporting Trials (CONSORT) Flow Diagram (see Supplementary Figure 2).

\section{Demographic and baseline variables}

A list of baseline measures for all participants are shown in Supplementary Table 1.

\section{Intervention compliance}

We will document the number of people who completed the different aspects of the intervention, including providing a saliva sample, successful genotyping, receipt of booklets and a phone call from the genetic counsellor.

\section{Efficacy analyses}

Efficacy analyses will be conducted on the basis of 'intention to treat'. All group comparisons will be twotailed with a nominal 5\% significance level, and will adjust for baseline scores and randomised stratification factors [40]. For (secondary) efficacy analyses that involve the pooled two-phenotype risk groups, the model will also include phenotypic risk (high, low).

\section{Primary efficacy analysis}

Geometric mean score and 95\% CI will also be represented graphically from over time (baseline, follow-ups 1 
Table 3 Domain-Specific Risk-Taking (DOSPERT) domain subscales

\begin{tabular}{ll}
\hline Domain subscale & Item text \\
\hline Health/safety & Drinking heavily at a social function \\
& Engaging in unprotected sex \\
& Driving a car without wearing a seat belt \\
& Riding a motorcycle without wearing a helmet \\
& Sunbathing without sunscreen \\
& Walking home alone at night in an unsafe area of \\
& town \\
& Admitting that your tastes are different from those \\
& of a friend \\
& Disagreeing with an authority figure on a major issue \\
Social & Choosing a career that you truly enjoy over a more \\
& prestigious one \\
& Speaking your mind about an unpopular issue in a \\
& meeting at work \\
& Moving to a city far away from your extended family \\
& Starting a new career in your mid-thirties
\end{tabular}

and 2). The primary analysis will be an intention-to-treat comparison of intervention and control arms for mean differences in UVR exposure measured as logtransformed daily SEDs at 12 months. The primary efficacy of the intervention will be assessed in each phenotypic risk group (high and low). UV dosimeter values will be log-transformed because of their rightskew distribution, and log-transformed values will be interpreted as a percentage change in the geometric mean of SEDs/day [41]. An analysis of covariance (ANCOVA), adjusted for baseline values and all randomised stratification factors will be performed [40]. A sensitivity analysis will be conducted on the subgroup of participants $(N=\sim 240)$ that will wear a UV dosimeter for 10 days at follow-up 1 (see subheading 'Sensitivity Analysis'); given the repeated nature of the outcomes in this subgroup, generalised linear mixed models (GLMMs) with random intercepts will be fitted to assess the overall intervention effect. The primary analysis will be based on the complete case principle given that the power was adjusted with a conservative loss to follow-up rate of $15 \%$. However, if $10 \%$ or more of the randomised patients do not have their primary outcome recorded, a sensitivity analysis will be performed using multiple imputation methods assuming that data are missing at random.

\section{Secondary efficacy analyses}

Where outcomes were assessed at three time points (baseline, 1 month, 12 months), including most questionnaire items, data will be analysed using GLMMs with random intercepts for continuous outcome measures, and generalised estimating equations (GEEs) with a log link function for binary outcome measures to estimate relative risks. The GLMM and GEE approach allows us to account for correlation due to repeated measures on each individual [42]. Where outcomes were assessed at two time points only (baseline and 12 months), data will be analysed using ANCOVA for continuous outcome measures [40], and using GLM with $\log$ link function for binomial outcome variables to estimate relative risks and 95\% CIs. The mean difference and $95 \%$ CIs between intervention and control groups at 12 months will be estimated.

\section{Exploratory efficacy analyses \\ Subgroup analysis}

The subgroup analyses will assess differences in intervention effects across pre-specified subgroups (see subheading 'Covariates and subgroups'). Tests of intervention effect modification will be performed by fitting intervention group and the relevant subgroup main effects and interaction into the models adjusted for baseline scores. Interpretation of evidence of heterogeneity of intervention effects among subgroups will remain exploratory (hypothesis generating) given that the study is not powered to test subgroups in the stratified analysis. Results will be presented as forest plots with $P$ values for heterogeneity (interaction test) for each pair of subgroups displayed.

\section{Sensitivity analysis}

A sensitivity analysis of the repeated measures UV dosimeter data will be conducted on the subgroup of participants $(N=\sim 240)$ who wear a UV dosimeter at 1 month and 12 months, using GLMMs with random intercepts to account for the repeated measures.

A sensitivity analysis will also be completed comparing the results for people who completed follow-up in the spring/summer of $2018 / 2019$ vs. $2019 / 2020$ due to the bushfires experienced in Australia during the latter period and the potential impact on personal and ambient UV exposure.

A sensitivity analysis will be conducted in which dosimeter data will be used to adjust for the effects of measurement error in the relative-risk estimate for self-reported sun exposure, using established methods [43].

\section{Technical details}

A second review statistician will independently reproduce the primary analyses. The reviewing statistician will have an overview of all the analyses and will check the code, producing tables selected at random as well as any other pieces of code as desired. 


\section{Supplementary information}

Supplementary information accompanies this paper at https://doi.org/10. 1186/s13063-020-04351-W

Additional file 1: Supplementary Figure 1. Trial schema.

Additional file 2: Supplementary Figure 2. Consolidated Standards of Reporting Trials (CONSORT) Flow Diagram.

Additional file 3: Supplementary Table 1. Demographic and baseline variables.

\section{Abbreviations}

Cl: Confidence interval; DOSPERT: Domain-specific Risk-taking; F1: Follow-up

1; F2: Follow-up 2; GEE: Generalised estimating equation; GLMM: Generalised linear mixed model; HEAP: Health Economics Analysis Plan; $\mathrm{ICH}$ E9: International Conference on Harmonisation, Statistical Principles for Clinical Trials; MAR: Missing at random; MHI-5: Mental Health Inventory; MICRA: Multidimensional Impact of Cancer Risk Assessment; RCT: Randomised controlled trial; SAP: Statistical analysis plan; SD: Standard deviation; SED: Standard Erythemal Dose; UV: Ultraviolet radiation

\section{Acknowledgements}

The study has been endorsed by the Melanoma and Skin Cancer Trials Group (ANZMTG 03.17). The authors and the authorship group would like to thank Bruce Armstrong for initial feedback on the study design, and Caro Badcock, Brooke Beswick, Georgina Fenton, Gillian Reyes-Marcelino, Ashleigh Sharman, Lauren Humphreys, Ainsley Furneaux-Bate, Rosa Evaquarta and Antigone Matsos for assistance with the conduct of this study. Managing Your Risk Study Group: Anne E Cust ${ }^{1,2}$, Ainsley J Newson ${ }^{3}$, Rachael L Morton ${ }^{4}$, Michael Kimlin ${ }^{5}$, Louise Keogh ${ }^{6}$, Matthew Law ${ }^{7}$, Judy Kirk ${ }^{8}$, Suzanne J Dobbinson ${ }^{9}$, Peter Kanetsky ${ }^{10}$, Graham Mann ${ }^{11}$, Hugh Dawkins ${ }^{12}$, Jacqueline Savard ${ }^{13}$, Kate Dunlop ${ }^{1}$, Lyndal Trevena', Mark Jenkins ${ }^{14}$, Martin Allen $^{15}$, Phyllis Butow ${ }^{16}$, Sarah Wordsworth ${ }^{17}$, Serigne Lo ${ }^{2}$, Cynthia Low ${ }^{18}$, Amelia K Smit ${ }^{1,2,3}$, David Espinoza ${ }^{4}$

\section{Authors' contributions}

SNL, AKS, DE and AEC wrote and finalised the SAP. All other authors provided input to the draft and read and approved the manuscript for publication. SL is the trial senior statistician, and AEC is the lead chief investigator for this trial.

\section{Funding}

This study is funded by the National Health and Medical Research Council of Australia (NHMRC; project grant 1129822). AEC receives a Career Development Fellowship from the NHMRC (1147843). AKS received a Research Training Program (RTP) Stipend Scholarship and a Merit Top Up Scholarship from the University of Sydney, and a Melanoma Institute Australia Postgraduate Research Scholarship. These funding bodies had no role in the design of the study and collection, analysis or interpretation of data or in writing the manuscript.

\section{Availability of data and materials}

Not applicable

\section{Ethics approval and consent to participate}

Ethical approval has been obtained from the Human Research Ethics Committee at The University of Sydney (2017/163). The study was carried out in compliance with national and state legal and regulatory requirements and according to the International Principles of Good Clinical Practice (ICHGCP). As such, all trial participants gave informed consent prior to joining the trial.

\section{Consent for publication}

Not applicable

\section{Competing interests}

The authors declare that they have no competing interests.

\section{Author details}

'The University of Sydney, Melanoma Institute Australia, Sydney, NSW, Australia. ${ }^{2}$ The University of Sydney, Faculty of Medicine and Health, Sydney School of Public Health, Cancer Epidemiology and Prevention Research, Sydney, NSW, Australia. ${ }^{3}$ The University of Sydney, Faculty of Medicine and Health, Sydney School of Public Health, Sydney Health Ethics, Sydney, NSW, Australia. ${ }^{4}$ The University of Sydney, NHMRC Clinical Trials Centre, Sydney, NSW, Australia.

Received: 19 February 2020 Accepted: 25 April 2020

Published online: 30 June 2020

\section{References}

1. Green AC, Williams GM, Logan V, Strutton GM. Reduced melanoma after regular sunscreen use: randomized trial follow-up. J Clin Oncol. 2011;29: 257-63.

2. Weinstock MA. Reducing death from melanoma and standards of evidence. J Invest Dermatol. 2012;132:1311-2.

3. Armstrong BK, Kricker A. How much melanoma is caused by sun exposure? Melanoma Res. 1993:3:395-401.

4. Kasparian NA, McLoone JK, Meiser B. Skin cancer-related prevention and screening behaviors: a review of the literature. J Behav Med. 2009;32:406-28.

5. Burton H, Chowdhury S, Dent T, Hall A, Pashayan N, Pharoah P. Public health implications from COGS and potential for risk stratification and screening. Nat Genet. 2013;45:349-51.

6. Pashayan N, Hall A, Chowdhury S, Dent T, Pharoah PD, Burton H. Public health genomics and personalized prevention: lessons from the COGS project. J Intern Med. 2013;274:451-6.

7. Glanz K, Rimer BK, Viswanath K. Health behavior. New York: Wiley; 2014.

8. McBride CM, Birmingham WC, Kinney AY. Health psychology and translational genomic research: bringing innovation to cancer-related behavioral interventions. Am Psychol. 2015;70:91-104.

9. Rogers RW. A protection motivation theory of fear appeals and attitude change. J Psychol. 1975;91:93-114.

10. Hollands GJ, French DP, Griffin SJ, Prevost AT, Sutton S, King S, Marteau TM. The impact of communicating genetic risks of disease on risk-reducing health behaviour: systematic review with meta-analysis. BMJ. 2016:352:11102.

11. Marteau TM, French DP, Griffin SJ, Prevost AT, Sutton S, Watkinson C, Attwood S, Hollands GJ. Effects of communicating DNA-based disease risk estimates on risk-reducing behaviours. Cochrane Database Syst Rev. 2010; (10):CD007275.

12. Smit AK, Newson AJ, Morton RL, Kimlin M, Keogh L, Law MH, Kirk J, Dobbinson S, Kanetsky PA, Fenton G, et al. The Melanoma Genomics Managing Your Risk Study: a protocol for a randomized controlled trial evaluating the impact of personal genomic risk information on skin cancer prevention behaviors. Contemp Clin Trials. 2018;70:106-16.

13. Smit AK, Espinoza D, Newson AJ, Morton RL, Fenton G, Freeman L, Dunlop $\mathrm{K}$, Butow PN, Law MH, Kimlin MG, et al. A pilot randomised controlled trial of the feasibility, acceptability and impact of giving information on personalised genomic risk of melanoma to the public. Cancer Epidemiol Biomarkers Prev. 2016. https://doi.org/10.1158/1055-9965.EPI-1116-0395.

14. Gamble C, Krishan A, Stocken D, Lewis S, Juszczak E, Dore C, Williamson PR, Altman DG, Montgomery A, Lim P, et al. Guidelines for the content of statistical analysis plans in clinical trials. JAMA. 2017;318:2337-43.

15. Dritsaki M, Gray A, Petrou S, Dutton S, Lamb SE, Thorn JC. Current UK practices on Health Economics Analysis Plans (HEAPs): are we using heaps of them? PharmacoEconomics. 2018;36:253-7.

16. Thieden E, Agren MS, Wulf HC. The wrist is a reliable body site for personal dosimetry of ultraviolet radiation. Photodermatol Photoimmunol Photomed. 2000;16:57-61.

17. Koster B, Sondergaard J, Nielsen JB, Allen M, Bjerregaard M, Olsen A, Bentzen J. Feasibility of smartphone diaries and personal dosimeters to quantitatively study exposure to ultraviolet radiation in a small national sample. Photodermatol Photoimmunol Photomed. 2015;31:252-60.

18. Dobbinson S, Niven P, Buller D, Allen M, Gies P, Warne C. Comparing handheld meters and electronic dosimeters for measuring ultraviolet levels under shade and in the sun. Photochem Photobiol. 2016;92:208-14.

19. Glanz K, Yaroch AL, Dancel M, Saraiya M, Crane LA, Buller DB, Manne S, O'Riordan DL, Heckman CJ, Hay J, Robinson JK. Measures of sun exposure and sun protection practices for behavioral and epidemiologic research. Arch Dermatol. 2008;144:217-22. 
20. Volkov A, Dobbinson SJ. 11 National Sun Protection Survey Report 2. Australians' sun protective behaviours and sunburn incidence on summer weekends, 2010-11 and comparison with 2003-04 and 2006-07. Melbourne: Centre for Behavioural Research in Cancer, Cancer Council Victoria; 2011 Oct. 2010.

21. Kasparian NA, Branstrom R, Chang YM, Affleck P, Aspinwall LG, Tibben A, Azizi E, Baron-Epel O, Battistuzzi L, Bruno W, et al. Skin examination behavior: the role of melanoma history, skin type, psychosocial factors, and region of residence in determining clinical and self-conducted skin examination. Arch Dermatol. 2012;148:1142-51.

22. Aspinwall LG, Taber JM, Kohlmann W, Leaf SL, Leachman SA. Perceived risk following melanoma genetic testing: a 2-year prospective study distinguishing subjective estimates from recall. J Genet Couns. 2014;23:421-37.

23. Cust AE, Armstrong BK, Smith BJ, Chau J, van der Ploeg HP, Bauman A. Selfreported confidence in recall as a predictor of validity and repeatability of physical activity questionnaire data. Epidemiology. 2009;20:43-441.

24. Aspinwall LG, Stump TK, Taber JM, Kohlmann W, Leaf SL, Leachman SA. Impact of melanoma genetic test reporting on perceived control over melanoma prevention. J Behav Med. 2015:38:754-65.

25. Djaja N, Youl P, Aitken J, Janda M. Evaluation of a skin self examination attitude scale using an item response theory model approach. Health Qual Life Outcomes. 2014;12:189.

26. Perez D, Kite J, Dunlop SM, Cust AE, Goumas C, Cotter T, Walsberger SC, Dessaix A, Bauman A. Exposure to the 'Dark Side of Tanning' skin cancer prevention mass media campaign and its association with tanning attitudes in New South Wales, Australia. Health Educ Res. 2015;30:336-46.

27. Marteau TM, Weinman J. Self-regulation and the behavioural response to DNA risk information: a theoretical analysis and framework for future research. Soc Sci Med. 2006;62:1360-8.

28. Smith SK, Simpson JM, Trevena LJ, McCaffery KJ. Factors associated with informed decisions and participation in bowel cancer screening among adults with lower education and literacy. Med Decis Mak. 2014;34(6):756-72.

29. Berwick DM, Murphy JM, Goldman PA, Ware JE Jr, Barsky AJ, Weinstein MC. Performance of a five-item mental health screening test. Med Care. 1991;29: 169-76

30. Peyre $\mathrm{H}$, Leplege A, Coste J. Missing data methods for dealing with missing items in quality of life questionnaires. A comparison by simulation of personal mean score, full information maximum likelihood, multiple imputation, and hot deck techniques applied to the SF-36 in the French 2003 decennial health survey. Qual Life Res. 2011;20:287-300.

31. Cella D, Hughes C, Peterman A, Chang CH, Peshkin BN, Schwartz MD, Wenzel L, Lemke A, Marcus AC, Lerman C. A brief assessment of concerns associated with genetic testing for cancer: the Multidimensional Impact of Cancer Risk Assessment (MICRA) questionnaire. Health Psychol. 2002;21:564-72.

32. DeMarco TA, Peshkin BN, Mars BD, Tercyak KP. Patient satisfaction with cancer genetic counseling: a psychometric analysis of the Genetic Counseling Satisfaction Scale. J Genet Couns. 2004;13:293-304.

33. McGivern B, Everett J, Yager GG, Baumiller RC, Hafertepen A, Saal HM. Family communication about positive BRCA1 and BRCA2 genetic test results. Genet Med. 2004;6:503-9.

34. Bentley AR, Callier S, Rotimi CN. Diversity and inclusion in genomic research: why the uneven progress? J Community Genet. 2017;8:255-66.

35. Treasure T, MacRae KD. Minimisation: the platinum standard for trials? Randomisation doesn't guarantee similarity of groups; minimisation does. BMJ. 1998:317:362-3.

36. Vuong K, Armstrong BK, Weiderpass E, Lund E, Adami H, Veierod MB, Barrett $J$, Davies JR, Bishop DT, Whiteman DC, et al. Development and external validation study of a melanoma risk prediction model based on selfassessed risk factors. JAMA Dermatol. 2016;152:9-6.

37. Blais AR, Weber EU. A Domain-Specific Risk-Taking (DOSPERT) scale for adult populations. Judgment Decis Making J. 2006;1:33-47.

38. Hay J, Kaphingst KA, Baser R, Li Y, Hensley-Alford S, McBride CM. Skin cancer concerns and genetic risk information-seeking in primary care. Public Health Genomics. 2012;15:57-72.

39. Morton RL, Asher R, Peyton E, Tran A, Smit AK, Butow PN, Kimlin MG, Dobbinson SJ, Wordsworth S, Keogh L, Cust AE. Risk attitudes and sun protection behaviour: can behaviour be altered by using a melanoma genomic risk intervention? Cancer Epidemiol. 2019;61:8-13.

40. European Medicines Agency Committee for Medicinal Products for Human Use (CHMP). Guideline on adjustment for baseline covariates in clinical trials.
2015. https://www.ema.europa.eu/en/adjustment-baseline-covariatesclinical-trials. Accessed 15 Jan 2020

41. Vittinghoff E, Glidden DV, Shiboski SC, McCulloch CE. Regression methods in biostatistics. New York: Springer-Verlag; 2012.

42. Toshiro T. Repeated measures design with generalized linear mixed models for randomized controlled trials. 1st ed. Boca Raton: Chapman \& Hall/CRC Biostatistics Series; 2017

43. White $E$, Armstrong BK, Saracci R. Principles of exposure measurement in epidemiology. 2nd ed. New York: Oxford University Press; 2008.

\section{Publisher's Note}

Springer Nature remains neutral with regard to jurisdictional claims in published maps and institutional affiliations.
Ready to submit your research? Choose BMC and benefit from:

- fast, convenient online submission

- thorough peer review by experienced researchers in your field

- rapid publication on acceptance

- support for research data, including large and complex data types

- gold Open Access which fosters wider collaboration and increased citations

- maximum visibility for your research: over $100 \mathrm{M}$ website views per year

At BMC, research is always in progress.

Learn more biomedcentral.com/submissions 